\title{
An Efficient Procedure for the Avoidance of Disconnected Incomplete Block Designs
}

\author{
J.D. Godolphin ${ }^{a, 1}$ and H.R. Warren ${ }^{b}$ \\ ${ }^{a}$ University of Surrey, Guildford, Surrey. GU2 7XH, UK \\ ${ }^{b}$ London School of Hygiene and Tropical Medicine, London, WC1E 7HT, UK
}

\begin{abstract}
Knowledge of the cardinality and the number of minimal rank reducing observation sets in experimental design is important information which makes a useful contribution to the statistician's tool-kit to assist in the selection of incomplete block designs. Its prime function is to guard against choosing a design that is likely to be altered to a disconnected eventual design if observations are lost during the course of the experiment. A method is given for identifying these observation sets based on the concept of treatment separation, which is a natural approach to the problem and provides a vastly more efficient computational procedure than a standard search routine for rank reducing observation sets. The properties of the method are derived and the procedure is illustrated by four applications which have been discussed previously in the literature.

Keywords: connectivity, incomplete block design, missing values, observation loss, rank reducing observation sets, robustness, selective partitioning
\end{abstract}

\footnotetext{
${ }^{1}$ Corresponding author: email j.godolphin@surrey.ac.uk
} 


\section{Introduction}

Experimental design researchers and practitioners have been aware for many years that serious field problems arise when one or more observations are not recorded, for whatever reason, during the experimental process so that the eventual design is different from the design that was planned originally. For instance, Yates (1933) drew attention to problems in the field "when the yields of some plots are lost, or are unreliable" and Cochran and Cox $(1957, \S 3.7)$ discussed difficulties "when certain observations are missing, through failure to record, or gross errors in recording or accidents". These experimenters were concerned primarily with the removal of orthogonality of the planned design but such concerns are mitigated in modern applications by routine use of statistical software packages. However, in experiments arranged in incomplete blocks, there are other problems with missing observations in the field that are not covered by data analysis packages, viz. (i) the inevitable loss in design efficiency and (ii) the risk that the eventual design is disconnected with respect to treatment effects, so that not all treatment contrasts are estimable. Dey et al. (2001) refer to these two problems as problems of design robustness efficiency and design robustness connectivity, respectively, and, of the two problems, it is the second which is easily the most serious. If the eventual design is disconnected the test of the usual null hypothesis that all treatment effects have the same value breaks down and many, if not most, of the pairwise treatment contrasts are inestimable. The avoidance of this unwelcome situation, through careful selection of the planned design, is clearly an important objective of both researcher and practitioner.

Computer-aided procedures for construction of incomplete block designs have been given by several authors, e.g. Mitchell (1974), Jones and Eccleston (1980), Whitaker et al. (1990), Nguyen and Miller (1992), John et al. (1993), Nguyen (1994), Angelis (2003), Soicher (2011) and others. The algorithms given by these authors employ various criteria for design construction, including a number of interchange and/or evolutionary techniques to search for an $A$-optimal design, either by maximizing the average efficiency factor, or some suitable approximation to it based on powers of the trace of the concurrence matrix. Since these methods do not appear to be programmed to cater for the problem of robustness connectivity, it is sensible to output several designs with

optimal or near-optimal properties and consider them for robustness before suggesting 
a design for experimental use. This strategy is reasonable because the most efficient design is not necessarily the best design according to robust connectivity criteria, see Godolphin (2004) and Bate et al. (2008), and it is good practice to compare robustness properties of the derived 'near-optimal' designs before making a final design selection.

It is assumed throughout this paper that it is not possible, realistically, to anticipate beforehand which observations are likely to go missing during an experiment. Godolphin (2004, 2006) refers to such observations as a rank-reducing observation set (RROS) that is Type 1 if the loss results in a disconnected eventual design and is Type 3 if the loss results in the elimination of all replicates of one or more treatments. The identification of RROSs by the approach of Godolphin (2006) makes use of the $Z$-matrix algebra of Theil (1965) and is a useful method if the design is not too large and the RROSs are small. However, the method is not practicable if the design is relatively large sized because of the large number of sets that require examination and it is not programmed to give information on which treatment contrasts are inestimable. This paper describes a procedure based on the concept of treatment separation, which is a two stage process that searches through a restricted class of subsets of the $v$ treatments and scans the blocks of the design to identify RROSs induced by these treatment subsets. This selective partitioning is a more informative approach to the identification of RROSs for incomplete block designs. Furthermore, when coupled with a method of adjusted selective partitioning it provides a procedure that is highly efficient computationally. The aim of the method is to find the smallest number of observations involved in the Type 1 RROSs and the number of such minimal sets; but the searching exercise is confined to relatively few treatment subsets so the output is obtained from far less computations than those necessary for a routine search through observation sets of increasing number and size.

The main results on selective partitioning and adjusted selective partitioning for the identification of RROSs of smallest cardinality are given in Section 2. Several properties of the method, with its implications for computation reduction, are derived. In Section 3 this procedure is illustrated by identifying the RROSs and examining the vulnerability of block designs in four experimental situations in the literature. The procedure is carried out using a program written in Matlab which can be found within the Online Supplementary Material here (insert link to online supplementary file here). 


\section{Description of the Procedure}

\subsection{Preliminaries}

Consider a planned connected binary block design, $D$, on $v$ treatments arranged in $b$ blocks of size $k(k<v)$; let $r_{i}$ be the number of replicates of the $i$ th treatment and let

$r_{[1]} \geq r_{[2]} \geq \ldots \geq r_{[v]}$ denote the treatment replication numbers in decreasing order. If one or more observations fail to be recorded during the course of the experiment then $D$ is effectively replaced by an eventual design $D_{e}$. In general, $D_{e}$ has $v_{*} \leq v$ treatments arranged in $b_{*} \leq b$ blocks of size varying between 1 and $k$. Either the $i$ th treatment does not occur in $D_{e}$ or it occurs in $D_{e}$ with replication number that is at most $r_{i}$. The difference between the two designs is the set of unavailable observations removed from $D$ to yield $D_{e}$ and this is referred to, simply, as an observation set.

\subsection{Rank Reducing Observation Sets}

The connectivity status of a block design is related directly to the rank of its design matrix: see, for example, Godolphin (2013). However, there are three kinds of rank reducing observation set (RROS), in which the rank of the design matrix for $D_{e}$ is strictly less than the rank of the design matrix for $D$.

(i) When a Type 1 RROS is removed from $D$ the eventual design $D_{e}$ is disconnected. In this case the $b_{*}$ blocks of $D_{e}$ are partitioned into two non-empty sets $S_{1}, S_{2}$ such that the blocks of $S_{1}$ contain all replicates of a proper subset of the $v_{*}$ treatments and the blocks of $S_{2}$ contain all replicates of the remaining treatments in $D_{e}$ : see Godolphin and Warren $(2011, \S 3)$ for further discussion of this situation. Equivalently, $D_{e}$ can be represented as a bipartite graph consisting of two sets of vertices, one set corresponding to the $v_{*}$ treatments and one set corresponding to the $b_{*}$ blocks, with an edge drawn between a treatment vertex and a block vertex whenever the treatment occurs in the block; this graph is disconnected since there is no path between any vertex for a treatment contained in blocks of $S_{1}$ and any vertex for a treatment contained in blocks of $S_{2}$.

(ii) A Type 2 RROS is any observation set which contains all observations from one or more whole blocks of $D$. A simple Type 2 RROS consists solely of the observations from a single block of $D$. There are $b$ simple Type 2 RROSs altogether with 
common cardinality $k$, and every Type 2 RROS contains one or more simple Type 2 RROSs. If the observation set lost from $D$ is a Type 2 RROS then $D_{e}$ has fewer blocks than $D$, i.e. $b_{*}<b$.

(iii) A Type 3 RROS is any observation set which contains the observations from all replicates of one or more of the $v$ treatments. A simple Type 3 RROS consists solely of the observations from all replicates of one treatment. There are $v$ simple Type 3 RROSs with cardinalities $r_{1}, r_{2}, \ldots, r_{v}$ and any simple Type 3 RROS corresponds to a simple Type 2 RROS in the dual of $D$, and conversely. Every Type 3 RROS contains one or more simple Type 3 RROSs and if a Type 3 RROS is lost from $D$ then fewer treatments occur in $D_{e}$ than in $D$, i.e. $v_{*}<v$.

Remark: In general, a RROS may be more than one type. Even a simple Type 2 RROS may also be a Type 1 RROS and, similarly, a simple Type 3 RROS may be a Type 1 RROS. This is demonstrated by the following design $D$ given by Cheng and Wu (1981), in which six treatments are arranged in seven blocks of size two.

\begin{tabular}{|c|c|c|c|c|c|c|c|}
\hline \multicolumn{8}{|c|}{ Blocks } \\
\hline & 1 & 2 & 3 & 4 & 5 & 6 & 7 \\
\hline Design D & {$\left[\begin{array}{ll}1 & 2\end{array}\right]$} & {$\left[\begin{array}{ll}2 & 3\end{array}\right]$} & {$\left[\begin{array}{ll}3 & 1\end{array}\right]$} & {$[45]$} & {$\left[\begin{array}{ll}5 & 6\end{array}\right]$} & {$\left[\begin{array}{ll}6 & 1\end{array}\right]$} & {$[46]$} \\
\hline
\end{tabular}

Fig 1: Cheng-Wu design $D$

It is clear that $D$ has seven simple Type 2 RROSs with cardinality $k=2$, and $D$ has six simple Type 3 RROSs with cardinalities $r_{1}=3, r_{2}=2, r_{3}=2, r_{4}=2, r_{5}=2$ and $r_{6}=3$. In particular, the observations in block 6 form a simple Type 2 RROS; and they also form a Type 1 RROS since their removal leaves a disconnected eventual design $D_{e}$ in which blocks 1, 2 and 3 contain all replicates of treatments labelled 1,2 and 3 whilst the remaining blocks 4,5 and 7 contain all replicates of treatments labelled 4,5 and 6. Similarly, the three observations corresponding to treatment labelled 1 in blocks 1, 3 and 6 form a simple Type 3 RROS; and they are a Type 1 RROS since the removal of these three observations leaves a disconnected eventual design in which the first three blocks contain all replicates of treatments labelled 2 and 3 and the remaining four blocks contain all replicates of treatments labelled 4,5 and 6. 
Definition: A Type 1 RROS containing $t \geq 1$ observations is a minimal Type 1 RROS (MRROS) if no Type 1 RROS contains fewer than $t$ observations. The number of distinct MRROSs is denoted by $T$.

This definition requires that a MRROS must be a Type 1 RROS. The following result shows that a MRROS cannot be a Type 2 RROS and it cannot be a Type 3 RROS.

Theorem 1. Every Type 1 RROS which is also a Type 2 RROS or a Type 3 RROS is not a MRROS.

Proof: Let $\theta$ be a Type 1 RROS which is removed from the design $D$. Then the blocks of the eventual design $D_{e}$ divide into two sets $S_{1}, S_{2}$ such that the blocks of $S_{1}$ have no treatments in common with the blocks of $S_{2}$.

Suppose firstly that $\theta$ is also a Type 2 RROS so that it includes one or more whole blocks of $D$, one of which is denoted by $B$. Keep $k-1$ observations of $B$ in the set $\theta$ but reinstate $B$ with just one observation so that a new eventual design, $D_{e 0}$ say, is obtained. Let $\theta_{0}$ be the observations in $D$ which are not in $D_{e 0}$ so the cardinality of $\theta_{0}$ is one less than that of $\theta$. Now all replicates of the single treatment in $B$ occur in the blocks of $S_{1}$ or of $S_{2}$, but not both, so add $B$ to that set. Then the blocks of $D_{e 0}$ are partitioned into two sets with no treatments in common, hence $\theta_{0}$ is a Type 1 RROS. It follows that $\theta$ is not a MRROS.

Suppose now that $\theta$ is a Type 1 RROS which is also a Type 3 RROS so that it includes all replicates of at least one treatment. Reinstate one replicate of this treatment so that a new eventual design, $D_{e 1}$ say, is obtained. As previously the observations in $D$ which are not in $D_{e 1}$ will have cardinality one less than that of $\theta$. Furthermore the blocks of $D_{e 1}$ are partitioned into the same sets $S_{1}, S_{2}$ such that the blocks of $S_{1}$ have no treatments in common with the blocks of $S_{2}$. Again it follows that $\theta$ is not a MRROS.

\subsection{Treatment Induced RROSs}

Treatment separation refers to the separation of the $v$ treatments of $D$ into nonempty sets $\phi$ and $\phi^{c}$. For definiteness it is assumed that the cardinality of $\phi$ is not greater than the cardinality of $\phi^{c}$. A treatment induced RROS refers to any observation set which is removed from $D$ to yield an eventual design having its blocks occupied by treatments from the set $\phi$ or the set $\phi^{c}$. 
Definition: A RROS is $\phi$-induced if each block of $D_{e}$ contains treatments either from $\phi$ only or from $\phi^{c}$ only. The class of all $\phi$-induced RROSs is denoted by $\Psi_{\phi}$.

Remark: Each member of $\Psi_{\phi}$ is a Type 1 RROS or a Type 3 RROS or both, and it can also be a Type 2 RROS. This is illustrated when $D$ is the Cheng-Wu design given in Fig. 1 and $\phi=\{1,2,3\}$. It is shown in $\S 2.2$ that the observations in block 6 form a Type 2 RROS which is a Type 1 RROS and is in $\Psi_{\phi}$. On the other hand the observations in block 1 form a Type 2 RROS which is not Type 1 nor Type 3 so it cannot be in $\Psi_{\phi}$. The two replicates of treatment 6 in blocks 5, 7 together comprise a Type 1 RROS but this is not in $\Psi_{\phi}$ since block 6 contains a treatment from $\phi$ and a treatment from $\phi^{c}$.

When a $\phi$-induced RROS is Type 1 the blocks of $D_{e}$ are partitioned into a set of blocks containing all replicates of treatments in $\phi$ and another set of blocks containing all replicates of treatments in $\phi^{c}$. However, if a $\phi$-induced RROS is Type 3 only then there is no partitioning of blocks since $D_{e}$ contains only treatments from $\phi$ or from $\phi^{c}$.

\subsection{Minimal partition numbers of a Design}

Whenever a MRROS is missing from $D$ then $D_{e}$ is disconnected and it follows from Theorem 1 that $b_{*}=b$ and $v_{*}=v$. The $v$ treatments of $D_{e}$ are separated into $\phi$ and $\phi^{c}$ and the $b$ blocks of $D_{e}$ are partitioned into two sets such that the $u$ treatments of $\phi\left(1 \leq u \leq\left\lfloor\frac{1}{2} v\right\rfloor\right)$ occur wholly within blocks of one set and the $v-u$ treatments of $\phi^{c}$ occur wholly within blocks of the other. The total number of estimable pairwise treatment contrasts is $\frac{1}{2} u(u-1)+\frac{1}{2}(v-u)(v-u-1)$ which may be substantially smaller than $\frac{1}{2} v(v-1)$, the total number of estimable pairwise treatment contrasts before any RROS is lost. If a simple Type 3 RROS is missing from $D$ then estimates of $v-1$ pairwise contrasts involving one treatment cannot be made so there are at most $\frac{1}{2}(v-1)(v-2)$ estimable pairwise treatment contrasts. Thus the loss of either a MRROS or a simple Type 3 RROS may be severely damaging to the aims of the experiment.

In practice, the simple Type 3 RROSs are noted merely by inspection of $D$. However, the MRROSs are not easy to identify, in general. The cardinality, $t$, of an MRROS and the total number, $T$, of the MRROSs are important properties of $D$ and, for simplicity, the terms $(t, T)$ are referred to as the minimal partition numbers of $D$.

In applications it is useful to have prior knowledge of both types of observation set 
so that the robustness of a design $D$ can be assessed before deciding to go ahead with an experiment using $D$. It is is important to know the value of $\tau$, the smaller of $t$ and $r_{[v]}$, since the largest cardinality of an observation set that cannot be a MRROS nor a Type 3 RROS is $\tau-1$. All pairwise treatment contrasts are estimable provided that no more than $\tau-1$ observations are lost during the experiment, irrespective of which observations these may be. This information is particularly helpful if $t<r_{[v]}$ since the robustness of the design to observation loss may be somewhat poor in this case and this will not be obvious by inspection of $D$.

It is assumed in this paper that each observation has the same chance of being lost during the experiment. Then the probability, $p_{\ell}$, that not all treatment contrasts will be estimable, conditional on the loss of $\ell$ observations, is dependent on the relative sizes of $t$ and $r_{[v]}$. If $\tau=t<r_{[v]}$, then $p_{\tau}=T /\left(\begin{array}{c}b k \\ \tau\end{array}\right)$; if $t>r_{[v]}=\tau$, then $p_{\tau}=N_{[v]} /\left(\begin{array}{c}b k \\ \tau\end{array}\right)$ where $N_{[v]}$ denotes the number of treatments with replication number $r_{[v]}$; and if $\tau=t=r_{[v]}$, then $p_{\tau}=\left(T+N_{[v]}\right) /\left(\begin{array}{c}b k \\ \tau\end{array}\right)$. Similar calculations apply for the probability $p_{\tau+1}$ that not all treatment contrasts will be estimable, conditional on the loss of $\tau+1$ observations, or the corresponding probabilities conditional on even greater observation loss.

If the minimal partition numbers are evaluated routinely at the planning stage of the experiment, they can assist the experimenter when choosing from several competing designs with the same values of $v, k, b$ and $r_{1}, \ldots, r_{v}$ and with the same or similar average efficiency factors. In this situation the robustness of the designs to loss of observations is a useful criterion upon which the final selection of $D$ may be based. The simple Type 3 RROSs for each of these designs have cardinalities $r_{1}, \ldots, r_{v}$ so only the minimal partition numbers need to be compared. The design with the largest MRROS cardinality is the suggested choice; and if two or more designs have this value of $t$ in common then the design with lowest value of $T$ is preferred. The minimal partition numbers can also assist when choosing from competing designs with different replication numbers, provided they are combined with the information on Type 3 RROSs for comparing the designs.

At present no theoretical method for identifying the MRROSs and for calculating the minimal partition numbers seems to be available. However, an algorithm for deriving the values of $(t, T)$ is described in the next section and the properties of the corresponding two-stage procedure are evaluated. 


\subsection{The SP-Process and ASP-Process}

For most large designs the task of examining all observation sets of reasonable cardinality to find those which are rank-reducing is prohibitive because of the very many sets involved. The method described here, based on the idea of treatment separation, is likely to be more useful since typically there are relatively few such separations even for a large design. Let the $v$ treatments of $D$ separate into disjoint sets $\phi$ and $\phi^{c}$ with cardinalities $u$ and $v-u$ respectively, where $1 \leq u \leq\left\lfloor\frac{1}{2} v\right\rfloor$. The Selective Partitioning process (SP-process) operates on $D$ to identify every RROS of smallest cardinality such that no pairwise treatment contrast between any treatment in $\phi$ and any treatment in $\phi^{c}$ is estimable. Suppose that $n_{h}$ is the number of blocks in $D$ containing exactly $h$ treatments in common with $\phi$. The SP-process operates block by block, using the following sequence of instructions:

For each block of D identify the observation set which consists of treatment entries in common with $\phi$, or in common with $\phi^{c}$, whichever is the smaller. If $k$ is even and there are $\frac{1}{2} k$ entries in the block in common with $\phi$ then either this set or the remaining set of entries in the block may be identified.

For a given $\phi$ the SP-process identifies all treatment entries of the following types:

(i) treatment entries from $\phi^{c}$ in blocks with $h>\frac{1}{2} k$;

(ii) treatment entries from $\phi$ in blocks with $h<\frac{1}{2} k$;

(iii) either treatment entries from $\phi$ only or $\phi^{c}$ only, in the particular case where $k$ is even, in blocks with $h=\frac{1}{2} k$.

It follows that for odd $k$, the SP-process generates a single RROS. For even $k$, the SP-process generates $2^{n_{k / 2}}$ RROSs corresponding to the $2^{n_{k / 2}}$ choices in (iii).

For a given $\phi$, the RROSs are all of size $\rho(\phi)$, where

$$
\rho(\phi)=\sum_{h=1}^{\lfloor(k-1) / 2\rfloor} h n_{h}+\sum_{h=\lfloor(k+1) / 2\rfloor}^{k-1}(k-h) n_{h} .
$$

Any set of $\rho(\phi)$ identified observations is a RROS belonging to the class of $\phi$-induced RROSs $\Psi_{\phi}$. The SP-process identifies RROSs with smallest cardinality amongst the many members of $\Psi_{\phi}$. It is of particular note that no Type 2 RROS is derived since no more than half of the entries of each block are identified by the SP-process. 


\section{Illustration: PBIB[2] Design T28}

To illustrate the use of the SP-process, consider the partially balanced design with two association classes (PBIB[2]) which is cited as T28 by Clatworthy (1973) in which ten treatments are arranged in five blocks of size four. The design is based on a triangular association scheme and is given in Fig. 2, where columns denote the five blocks.

\section{$\underline{\text { Blocks }}$}

\begin{tabular}{ccccc}
$\mathbf{1}$ & $\mathbf{2}$ & $\mathbf{3}$ & $\mathbf{4}$ & $\mathbf{5}$ \\
\hline 1 & 1 & 2 & 3 & 4 \\
2 & 5 & 5 & 6 & 7 \\
3 & 6 & 8 & 8 & 9 \\
4 & 7 & 9 & 10 & 10 \\
\hline
\end{tabular}

Fig 2: Partially balanced triangular design T28

Consider $u=1$ : there are no Type 1 RROSs identified by the SP-process so there is no block partition, however the SP-process gives rise to ten Type 3 RROSs of cardinality two by identifying both replicates of a single treatment. An eventual design is shown in Fig. 3 (i) with $\phi=\{3\}$, where symbol ' $*$ ' denotes a missing observation that is removed to the RROS. This simple Type 3 RROS, showing both replicates of treatment 3 removed from $D$, is the only $\{3\}$-induced RROS generated by the SP-process. Two disconnected designs separating treatment 3 from the other nine treatments, after partitioning the five blocks into sets $S_{1}, S_{2}$, are given by Fig. 3 (ii), (iii), however these eventual designs cannot be obtained from the SP-process in its present form.

(i) no block partition

i) \begin{tabular}{ccccc}
$\mathbf{1}$ & $\mathbf{2}$ & $\mathbf{3}$ & $\mathbf{4}$ & $\mathbf{5}$ \\
\hline 1 & 1 & 2 & $*$ & 4 \\
2 & 5 & 5 & 6 & 7 \\
$*$ & 6 & 8 & 8 & 9 \\
4 & 7 & 9 & 10 & 10 \\
\hline
\end{tabular}

\begin{tabular}{|c|c|c|c|c|}
\hline$S_{1}$ & & & $S_{2}$ & \\
\hline 1 & 2 & 3 & 4 & 5 \\
\hline (ii) & 1 & 2 & $*$ & 4 \\
\hline$*$ & 5 & 5 & 6 & 7 \\
\hline 3 & 6 & 8 & 8 & 9 \\
\hline * & 7 & 9 & 10 & 10 \\
\hline
\end{tabular}

\begin{tabular}{|c|c|c|c|c|c|}
\hline & $S_{1}$ & & & $\gamma_{2}$ & \\
\hline & 4 & 1 & 2 & 3 & 5 \\
\hline (iii) & 3 & 1 & 1 & 2 & 4 \\
\hline & $*$ & 2 & 5 & 5 & 7 \\
\hline & $*$ & $*$ & 6 & 8 & 9 \\
\hline & $*$ & 4 & 7 & 9 & 10 \\
\hline
\end{tabular}

Fig 3: Eventual designs after (i) SP-process and (ii), (iii) ASP-process with $\phi=\{3\}$

For $u=2$, two types of RROS are generated by the SP-process and examples of eventual designs are shown in Fig. 4 for (i) $\phi=\{1,2\}$ and (ii) $\phi=\{1,8\}$, where $\rho(\phi)=4$. Fig. 4 (i) shows that a Type 1 RROS is obtained which partitions blocks into $S_{1}=\{$ block 1$\}$ and $S_{2}=\{$ blocks $2,3,4,5\}$ and separates the treatments into $\phi=\{1,2\}$ and $\phi^{c}=\{3, \ldots, 10\}$. 
There are $2^{n_{k / 2}}=2^{1}=2$ RROSs which are $\{1,2\}$-induced RROSs generated by the SPprocess and the other, not displayed here, is Type 3. Fig. 4 (ii) shows that the only $\{1,8\}$-induced RROS generated by the SP-process is Type 3 but not Type 1 . A RROS which separates treatments 1 and 8 from the other treatments is given in Fig. 4 (iii); this RROS is Type 1 and Type 3, since both replicates of treatment 2 are missing, however it is not obtained from the SP-process in its present form.

(i)

\begin{tabular}{ccccc}
$S_{1}$ & \multicolumn{5}{c}{$S_{2}$} \\
$\mathbf{1}$ & $\mathbf{2}$ & $\mathbf{3}$ & $\mathbf{4}$ & $\mathbf{5}$ \\
\hline 1 & $*$ & $*$ & 3 & 4 \\
2 & 5 & 5 & 6 & 7 \\
$*$ & 6 & 8 & 8 & 9 \\
$*$ & 7 & 9 & 10 & 10 \\
\hline
\end{tabular}

\begin{tabular}{|c|c|c|c|c|}
\hline 1 & 2 & 3 & 4 & 5 \\
\hline$*$ & $*$ & 2 & 3 & 4 \\
\hline 2 & 5 & 5 & 6 & 7 \\
\hline 3 & 6 & $*$ & $*$ & 9 \\
\hline 4 & 7 & 9 & 10 & 10 \\
\hline
\end{tabular}

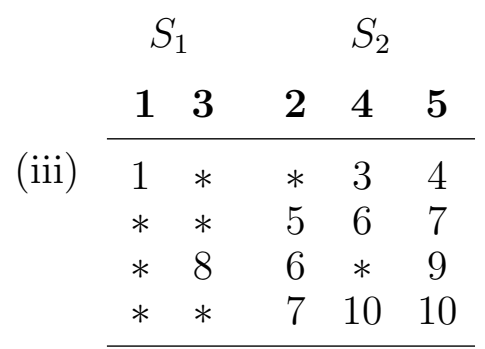

Fig 4: Eventual designs after SP-process with (i) $\phi=\{1,2\}$, (ii) $\phi=\{1,8\}$ and after ASP-process with (iii) $\phi=\{1,8\}$

Theorem 2. Let $\phi$ be a set of $u$ treatments of $D,\left(1 \leq u \leq\left\lfloor\frac{1}{2} v\right\rfloor\right)$. Every RROS belonging to the $\phi$-induced set $\Psi_{\phi}$ consisting of $\rho(\phi)$ observations is generated by the SPprocess. Furthermore, no RROS belonging to $\Psi_{\phi}$ consists of fewer than $\rho(\phi)$ observations.

The proof of Theorem 2 is considered after the statement and proof of Theorem 3 . This result shows that for given $\phi$, the SP-process can generate Type 1 RROSs of minimum cardinality, separating $\phi$ and $\phi^{c}$, in a natural and efficient manner. However the SP-process may also give RROSs that are Type 3 only, as illustrated by design T28. Indeed for any $\phi$ with $u<\frac{1}{2} k$ the SP-process always generates Type 3 RROSs that give eventual designs containing treatments just from the complementary treatment set $\phi^{c}$, as seen in Fig. 3 (i) where there is no block partition. Furthermore, other Type 3 RROSs sometimes arise with $u \geq \frac{1}{2} k$, as seen in Fig. 4 (ii). Since it is essential that Type 3 RROSs are not confused with the Type 1 RROSs, an adjustment of the SP-process is needed to ensure that each eventual design contains a replicate of every treatment in $\phi$, and this depends on the notion of lowest covering.

Definition: A collection of blocks which together contain at least one replicate of each treatment in $\phi$ is a covering for $\phi$. The weight of the covering is defined as

$$
\sum_{h=1}^{\lfloor(k-1) / 2\rfloor}(k-2 h) m_{h}
$$


where $m_{h}$ is the number of blocks in the covering that contain $h$ treatments from $\phi$. A covering for $\phi$ in which the weight is minimized is a lowest covering for $\phi$, denoted $\Gamma$, and the minimum value of the weight is denoted by $\gamma(\phi)$.

To ensure that at least one replicate of each member of $\phi$ will be in $D_{e}$, the SP-process is adjusted so that observations identified for inclusion in the RROS from blocks of a lowest covering are exactly those treatments from $\phi^{c}$. The Adjusted Selective Partitioning process (ASP-process) operates block by block, using the following procedure:

For each block of a lowest covering of $\phi$, identify the observation set which consists of treatment entries in $\phi^{c}$ only, i.e. do not identify any of the entries in $\phi$. For the remaining blocks of D, employ the SP-process.

For a given $\phi$ and $\Gamma$, the ASP-process identifies all treatment entries of the following types:

(i) treatment entries from $\phi^{c}$ in blocks in the covering;

(ii) treatment entries from $\phi^{c}$ from blocks with $h>\frac{1}{2} k$ not in the covering;

(iii) treatment entries from $\phi$ from blocks with $h<\frac{1}{2} k$ not in the covering;

(iv) either treatment entries from $\phi$ only or $\phi^{c}$ only, in the particular case where $k$ is even, in blocks with $h=\frac{1}{2} k$ which are not in the covering.

For the given covering, the ASP-process generates a single RROS for odd $k$ and up to $2^{n_{k / 2}}$ RROSs, corresponding to the choices in (iii), for even $k$.

The RROSs are all of size $\rho_{A}(\phi)$, where

$$
\rho_{A}(\phi)=\rho(\phi)+\gamma(\phi)
$$

As with the SP-process, a set of observations identified by the ASP-process is a RROS by construction. The use of a lowest covering $\Gamma$ ensures that $D_{e}$ includes each treatment from $\phi$. The ASP-process separates treatments in $\phi$ from those in $\phi^{c}$, either by generating a Type 1 RROS via a block partition or by generating a Type 3 RROS consisting of all replicates of treatments from $\phi^{c}$. In particular, no Type 3 RROS which includes all replicates of one or more treatments from $\phi$ is generated by the ASP-process.

\section{Illustration (Continued): PBIB[2] Design T28}

Use of the ASP-process on $\phi=\{3\}$ gives $\rho_{A}(\phi)=\rho_{A}(\{3\})=\rho(\phi)+\gamma(\phi)=4$. One RROS is identified as Type 1 and is shown in Fig. 3 (ii) with lowest covering $\Gamma$ given by 
the single block 1. Another Type 1 RROS is shown in Fig. 3 (iii) with lowest covering being the single block 4 .

Use of the ASP-process on $\phi=\{1,8\}$ gives $\rho_{A}(\phi)=\rho_{A}(\{1,8\})=\rho(\phi)+\gamma(\phi)=8$. The RROS identified is Type 1 and Type 3, and it is displayed in Fig. 4 (iii) with lowest covering comprising blocks 1 and 3 .

Theorem 3. Let $\phi$ be a set of $u$ treatments of $D,\left(1 \leq u \leq\left\lfloor\frac{1}{2} v\right\rfloor\right)$, and consider the set of RROSs $\Psi_{\phi 0} \subset \Psi_{\phi}$ for which the corresponding eventual designs contain every treatment in $\phi$ replicated at least once. Every RROS belonging to $\Psi_{\phi 0}$ consisting of $\rho_{A}(\phi)$ observations is generated by the ASP-process. Furthermore, no RROS belonging to $\Psi_{\phi 0}$ consists of fewer than $\rho_{A}(\phi)$ observations.

Proof: Consider a RROS $\psi \in \Psi_{\phi 0}$. Each block of $D_{e}$ contains treatments either from $\phi$ or from $\phi^{c}$, but not both, so let $S_{1}$ denote the set of blocks containing treatments from $\phi$ and let $S_{2}$ denote the remaining blocks of $D_{e}$. From the conditions of the theorem it is possible to choose a subset, $S_{1 \psi}$ say, of $S_{1}$ such that every treatment in $\phi$ is replicated at least once in the blocks of $S_{1 \psi}$.

Now consider the same set $S_{1 \psi}$ of blocks of the design $D$. By definition, these blocks provide a covering for $\phi$; and $m_{h}$ of these blocks contain exactly $h$ treatments in common with $\phi$ and $k-h$ treatments in common with $\phi^{c}(h=1, \ldots, k)$. The observations with treatments in common with $\phi^{c}$ must be in $\psi$, i.e. the blocks of $S_{1 \psi}$ contribute

$$
\sum_{h=1}^{k}(k-h) m_{h}+n_{c o v}
$$

observations to $\psi$, where $n_{\text {cov }} \geq 0$ is the number of observations with treatments in common with $\phi$ that are included in $\psi$ but would not be identified by the ASP-process.

The remaining blocks of $D$ not in the covering may also contribute some observations to $\psi$. If such a block has exactly $h$ treatments in common with $\phi$, the total number of observations included in $\psi$ is at least as large as the smaller of $h$ and $k-h$. Therefore, the number of observations that blocks not in the covering contribute to $\psi$ is

$$
\sum_{h=1}^{\lfloor(k-1) / 2\rfloor} h\left(n_{h}-m_{h}\right)+\sum_{h=\lfloor(k+1) / 2\rfloor}^{k-1}(k-h)\left(n_{h}-m_{h}\right)+n_{\text {noncov }}
$$

where $n_{\text {noncov }} \geq 0$ is the number of observations that are included in $\psi$ from the blocks not in the covering that would not be identified by the ASP-process. Hence the total 
number of observations in $\psi$ is at least

$$
\begin{aligned}
\sum_{h=1}^{\lfloor(k-1) / 2\rfloor} h n_{h} & +\sum_{h=\lfloor(k+1) / 2\rfloor}^{k-1}(k-h) n_{h}+\sum_{h=1}^{[(k-1) / 2]}(k-2 h) m_{h} \\
& =\rho(\phi)+\sum_{h=1}^{[(k-1) / 2]}(k-2 h) m_{h} \geq \rho(\phi)+\gamma(\phi)=\rho_{A}(\phi),
\end{aligned}
$$

from (2.2). It follows that $\psi$ contains at least $\rho_{A}(\phi)$ observations. Note that equality occurs in (2.3) if the set of blocks of $D$ given by $S_{1 \psi}$ is a lowest covering. In this case a necessary and sufficient condition for $\psi$ to contain $\rho_{A}(\phi)$ observations is that $n_{\text {cov }}=n_{\text {noncov }}=0$, however this occurs if observations are identified in blocks of $D$ in accordance with the ASP-process. This proves the theorem.

It is evident that Theorem 2 can be established by the same argument, except that a covering is not involved, and details of the proof are omitted.

\subsection{Identification of MRROSs}

The SP-process and ASP-process suggest an approach to identifying MRROSs by considering all possible separations of the $v$ treatments into non-empty sets $\phi$ and $\phi^{c}$, then identifying the corresponding RROSs and choosing Type I sets of smallest cardinality. A difficulty with this approach is that Type 3 RROSs may be encountered and it is necessary to be assured that this will not obscure identification of any of the MRROSs when applying the method. This point is pivotal and is considered now. It is first shown that not all separations of the $v$ treatments into sets $\phi$ and $\phi^{c}$ need be considered when applying the method. In particular, if $D$ has treatments from $\phi$ occupying less than half of the entries in each block it is possible that no MRROS exists for this treatment separation, depending on lowest covering structure, as stated in the following lemma.

Lemma 1. Let $D$ be a block design on $v$ treatments in blocks of size $k$. Suppose $\phi$ is a subset comprising $u$ of the treatments of $D$, with $1 \leq u \leq\left\lfloor\frac{1}{2} v\right\rfloor$, such that the following two conditions are satisfied:

(i) $n_{h}=0$ for all $h \geq\left\lfloor\frac{1}{2}(k+1)\right\rfloor$;

(ii) a lowest covering comprises at least two blocks.

Then no MRROS exists which partitions the $v$ treatments into sets $\phi$ and $\phi^{c}$.

Proof: Condition (i) implies that there are more treatments from $\phi^{c}$ than from $\phi$ in each block of $D$. Let $\Gamma$ be a lowest covering for $\phi$, which consists of at least two blocks, 
and let $B$ be a block of $\Gamma$. Denote the number of treatments from $\phi$ in $B$ by $u_{B}$ and let the set of these treatments be $\phi_{B}$. From (ii) each block of $\Gamma$ contains a proper subset of $\phi$, hence $u_{B}<u$. Also, from (i) every block in $\Gamma$ makes a strictly positive contribution to $\gamma(\phi)$ and $B$ is itself a lowest covering for $\phi_{B}$, therefore $\gamma(\phi)>\gamma\left(\phi_{B}\right)$. Furthermore, from (i), (2.1) simplifies to give

$$
\rho(\phi)=\sum_{i \in \phi} r_{i} \text { and } \rho\left(\phi_{B}\right)=\sum_{i \in \phi_{B}} r_{i},
$$

i.e. $\rho(\phi)>\rho\left(\phi_{B}\right)$ and it follows from (2.2) that $\rho_{A}(\phi)>\rho_{A}\left(\phi_{B}\right)$. The observations identified using the ASP-process on $\phi_{B}$, with lowest covering block $B$, are the treatment entries from $\phi_{B}^{c}$ in $B$ and from $\phi_{B}$ in the remaining blocks of $D$. Therefore, when this RROS is removed from $D$ the blocks of the eventual design are partitioned into $S_{1}$, consisting solely of block $B$, and $S_{2}$, the set of all the other blocks. Thus the RROS is Type 1 and $t \leq \rho_{A}\left(\phi_{B}\right)<\rho_{A}(\phi)$. It follows from Theorems 1 and 3 that no MRROS exists which separates the $v$ treatments into sets $\phi$ and $\phi^{c}$.

When the treatment separation $\phi$ and $\phi^{c}$ is such that $u$ is relatively large, there is an interesting and useful corollary of Lemma 1 which establishes that if all RROSs that are generated by the SP-process are Type 3 only then no MRROSs exist for this particular treatment separation.

Lemma 2. Let $\phi$ be a set of $u$ treatments of $D$, where $\left\lfloor\frac{1}{2}(k+1)\right\rfloor \leq u \leq\left\lfloor\frac{1}{2} v\right\rfloor$. Suppose every RROS identified by the SP-process operating on $\phi$ includes either: all replicates of every treatment from $\phi$; or all replicates of every treatment from $\phi^{c}$. Then no MRROS exists which separates the $v$ treatments into sets $\phi$ and $\phi^{c}$.

Proof: Block-size $k=2$ is not included since the SP-process must generate some Type 1 RROSs in this case and, therefore, the conditions of the lemma cannot apply. Let $k \geq 3$ and suppose that every RROS identified by the SP-process includes all replicates of every treatment from $\phi$. Then $n_{h}=0$ for all $h \geq\left\lfloor\frac{1}{2}(k+1)\right\rfloor$. Also, any lowest covering of $\phi$ contains at least two blocks since $u \geq\left\lfloor\frac{1}{2}(k+1)\right\rfloor$. Thus, Lemma 1 applies and the result follows. The proof of the remaining part of the lemma follows similarly.

These two lemmas imply that the SP- and ASP-processes can be used for identifying all MRROSs. For large $u$ the computational problems of formulating lowest coverings is not an issue since the SP-process alone is sufficient for this identification, as established in the following result. 
Theorem 4. Every MRROS for design D arises from either one or both of the following: (i) use of the ASP-process with $u \in\left\{1, \ldots,\left\lfloor\frac{1}{2}(k-1)\right\rfloor\right\}$; (ii) use of the SP-process with $\left.u \in\left\{\left\lfloor\frac{1}{2}(k+1)\right\rfloor\right\}, \ldots,\left\lfloor\frac{1}{2} v\right\rfloor\right\}$.

Proof: Suppose that a MRROS exists for the treatment separation $\phi$ and $\phi^{c}$, where $\phi$ is a subset of $u$ treatments with $u \in\left\{1, \ldots,\left\lfloor\frac{1}{2}(k-1)\right\rfloor\right\}$. Lemma 1 implies that every lowest covering for $\phi$ is a single block. Let $B$ be such a block; then the ASP-process generates a Type 1 RROS, $\theta$ say, with treatments from $\phi^{c}$ in $B$ and treatments from $\phi$ in the other $b-1$ blocks. By Theorem 3 no Type 1 RROS with smaller cardinality exists, hence $\theta$ is a MRROS. Also by Theorem 3, every RROS of the same cardinality for the treatment separation $\phi$ and $\phi^{c}$ is generated by the ASP-process, i.e. (i) follows since all MRROSs separating $\phi$ and $\phi^{c}$ are identified by the method.

Now suppose that a MRROS exists for the treatment separation $\phi$ and $\phi^{c}$, where $\phi$ is a subset of $u$ treatments with $\left.u \in\left\{\left\lfloor\frac{1}{2}(k+1)\right\rfloor\right\}, \ldots,\left\lfloor\frac{1}{2} v\right\rfloor\right\}$. Lemma 2 shows that at least one Type 1 RROS, $\theta$ say, with this separation is identified by the SP-process. By Theorem 2 no Type 1 RROS with smaller cardinality exists for this treatment separation, so $\theta$ is a MRROS. Theorem 2 also shows that all MRROSs for this treatment separation are identified by the method. This shows (ii) that the SP-process generates the MRROSs for larger treatment sets.

\section{Illustration (Continued): PBIB[2] Design T28}

The first stage of the procedure involves the ASP-process when $u=1$. Each treatment is held isolated in a single block, separated from the others which occur in the remaining four blocks, on two occasions each: illustrations of two such eventual designs are given by Fig. 3 (ii) and (iii). This gives 20 RROSs of cardinality $\rho_{A}(\phi)=4$. The second stage involves the SP-process when $u=2,3,4$. This stage gives a further 55 Type 1 RROSs of cardinality 4 . One illustration of such an eventual design with $u=2$ is given by Fig. 4 (i). Hence $(t, T)=(4,75)$ for design T28.

\subsection{Implementation of the Procedure}

Theorem 4 establishes that the MRROSs separating treatments of $D$ into sets $\phi, \phi^{c}$ are obtained in two stages, viz. Stage 1 for all $\phi$ with $1 \leq u \leq\left\lfloor\frac{1}{2}(k-1)\right\rfloor$ using the ASP-process, and Stage 2 for all $\phi$ with $\left\lfloor\frac{1}{2}(k+1)\right\rfloor \leq u \leq\left\lfloor\left(\frac{1}{2} v\right)\right\rfloor$, using the SP-process. The search in Stage 2 is straightforward and generates $2^{n_{k / 2}}$ RROSs of size $\rho(\phi)$ for each 
$\phi$. It appears at first sight that Stage 1 is more computer intensive, since the lowest coverings are required for each $\phi$. However, Theorem 5 and Corollaries 1 and 2 show that the results of the ASP-process can be obtained in closed form without a formal scan. These results are dependent only on the values of the two smallest replication numbers $r_{[v]}$ and $r_{[v-1]}$, together with the numbers and locations of treatments occurring in $D$ no more than twice, subject to the mild condition that either $r_{[v]} \geq 2$ or $r_{[v-1]} \geq 3$.

Theorem 5. Suppose that $D$ is such that either $r_{[v]} \geq 2$ or $r_{[v-1]} \geq 3$. Let $\phi$ denote a set of $u$ treatments of $D$, with $1 \leq u \leq\left\lfloor\frac{1}{2}(k-1)\right\rfloor$, and let $\Phi$ be the set of all such $\phi$. Further, let $t_{1}$ denote the smallest number of observations in a Type 1 RROS that separates at least one member of $\Phi$ from its complement. Then the value of $t_{1}$ depends on $r_{[v]}$ and $r_{[v-1]}$ as follows:

(i) if $r_{[v-1]} \geq 3$ then $t_{1}=k+r_{[v]}-2$;

(ii) if $r_{[v]}=r_{[v-1]}=2$ then $t_{1}=k$.

Proof: Let $\Phi_{0} \subset \Phi$ be the subset whose members have lowest covering comprising a single block. By a similar argument to that used in Lemma 1 , to identify $t_{1}$ attention is restricted to $\phi \in \Phi_{0}$. For such $\phi,(2.2)$ simplifies to:

$$
\rho_{A}(\phi)=k+\sum_{i \in \phi}\left(r_{i}-2\right) .
$$

For $\phi \in \Phi_{0}$ the RROS identified by the ASP-process is Type 1 since the block of a lowest covering is the only member of $S_{1}$ in the eventual design and the other $b-1$ blocks form the set $S_{2}$. Thus by Theorem 3

$$
t_{1}=\min _{\phi \in \Phi_{0}}\left\{\rho_{A}(\phi)\right\}=\min _{\phi \in \Phi_{0}}\left\{k+\sum_{i \in \phi}\left(r_{i}-2\right)\right\} .
$$

For given $u \in\left\{1, \ldots,\left\lfloor\frac{1}{2}(k-1)\right\rfloor\right\}$, the least possible value of $\rho_{A}(\phi)$ occurs if $\phi$ is a set of treatments with replications $r_{[v]}, \ldots, r_{[v-u+1]}$ that appear in a single block. Thus:

$$
\rho_{A}(\phi) \geq k+\sum_{i=1}^{u}\left(r_{[v-i+1]}-2\right) .
$$

The lower bound in (2.6) is realized for $u=1$ when $\phi$ is any treatment with replication $r_{[v]}$, giving $t_{1} \leq k+r_{[v]}-2$. So consider a set of treatments $\phi \in \Phi_{0}$ with cardinality $2 \leq u \leq\left\lfloor\frac{1}{2}(k-1)\right\rfloor$. An element of $\phi$ with replication 3 or more makes a positive contribution to $\rho_{A}(\phi)$, elements of $\phi$ with replication 2 make zero contribution and elements of $\phi$ with replication 1 make a negative contribution. Thus: 
(i) If $r_{[v-1]} \geq 3$, any $\phi$ with $u>1$ has $\rho_{A}(\phi)>k+r_{[v]}-2$. Therefore $t_{1}=k+r_{[v]}-2$ and any RROS which separates the treatments into $\phi, \phi^{c}$ with $u \leq\left\lfloor\frac{1}{2}(k-1)\right\rfloor$ has $\phi$ comprising a single treatment with replication $r_{[v]}$.

(ii) If $r_{[v]}=r_{[v-1]}=2$, then $t_{1}=k$ since any $\phi$ comprising treatments with replication 2 contained in a single block has $\rho_{A}(\phi)=k$. Any $\phi$ involving a treatment with replication 3 or more gives $\rho_{A}(\phi)>k$.

Corollary 1. Suppose that $D$ is such that either $r_{[v]} \geq 2$ or $r_{[v-1]} \geq 3$. The total number, $T_{1}$, of Type 1 RROSs of size $t_{1}$ which correspond to treatment separation $\phi, \phi^{c}$ for $\phi \in \Phi$ is given by the two cases below.

(i) If $r_{[v-1]} \geq 3$, then $T_{1}=N_{[v]} r_{[v]}$, where $N_{[v]}$ is the number of treatments with replication $r_{[v]}$.

(ii) If $r_{[v]}=r_{[v-1]}=2$, denote the number of blocks containing treatments with replication 2 by $p$ and the number of treatments with replication 2 in these blocks by $\beta_{1}, \beta_{2}, \ldots, \beta_{p}$ and let $\delta_{i}=\min \left\{\beta_{i},\left\lfloor\frac{1}{2}(k-1)\right\rfloor\right\}$. Then $T_{1}=\sum_{i=1}^{p} \sum_{j=1}^{\delta_{i}}\left(\begin{array}{c}\beta_{i} \\ j\end{array}\right)$.

The results in Corollary 1 follow in a straightforward way from Theorem 5 since, for each $\phi \in \Phi$ that yields Type 1 RROSs of size $t_{1}$, each lowest covering of $\phi$ gives rise to exactly one such RROS. The details are omitted.

Remark: For completeness it is required to consider the unlikely situation in which the condition of Theorem 5 and Corollary 1 is not satisfied, i.e. $r_{[v]}=1$ and $r_{[v-1]} \leq 2$.

Corollary 2. Suppose that $D$ is such that $r_{[v]}=1$ and $r_{[v-1]} \leq 2$. Let $\Phi$ and $t_{1}$ be as in Theorem 5. Then $t_{1}=k-\alpha$, where $\alpha$ denotes either the largest number of treatments with replication unity contained in a single block or the number $\left\lfloor\frac{1}{2}(k-1)\right\rfloor$, whichever is the smaller. Further, let the number of blocks containing $\alpha$ treatments with replication 1 be $q$, let the number of treatments with replication 2 in these blocks be $\beta_{1}, \beta_{2}, \cdots, \beta_{q}$ and let $\delta_{i}=\min \left\{\beta_{i},\left\lfloor\frac{1}{2}(k-1)\right\rfloor-\alpha\right\}$. Then $T_{1}=\sum_{i=1}^{q} \sum_{j=0}^{\delta_{i}}\left(\begin{array}{c}\beta_{i} \\ j\end{array}\right)$, where $T_{1}$ is the total number of Type 1 RROSs of size $t_{1}$ given in Corollary 1.

Proof: Let $\phi$ be any set consisting of $\alpha$ treatments with replication unity and as many as $\left\lfloor\frac{1}{2}(k-1)\right\rfloor-\alpha$ treatments with replication 2 , all in a single block. From the argument of Theorem $5, \rho_{A}(\phi)=k-\alpha=t_{1}$. Also, any such $\phi$ gives rise to exactly one Type 1 RROS of size $k-\alpha$ and the value for $T_{1}$ follows.

The procedure is in three stages. Stage 1 obtains $t_{1}$ by Theorem 5 . For designs with $k=2$ or with $\alpha \geq\left\lfloor\frac{1}{2}(k+1)\right\rfloor$, MRROSs only arise from Stage 2 . In these cases $t_{1}$ is set large enough to ensure all MRROSs from Stage 2 are recorded. Stage 2 involves a 
scan of the blocks of $D$ for each $\phi$ with $\left\lfloor\frac{1}{2}(k+1)\right\rfloor \leq u \leq\left\lfloor\frac{1}{2}(v)\right\rfloor$ using the SP-process. Any $\phi$ with $\rho(\phi) \leq t_{1}$ are saved and assessed in increasing order of $\rho(\phi)$ to determine the nature of the RROSs generated. If any $\phi$ with $\rho(\phi) \leq t_{1}$ exist for which the SP-process generates at least one Type 1 RROS, then $t_{2}$ is defined as the smallest size of such a RROS. Any Type 1 RROS of this size from Stage 2 is an MRROS and is saved. Stage 3 combines the results from Stages 1 and 2 and outputs $t$ and the $T$ MRROSs.

\section{Procedure to Identify MRROSs}

Input: design $D$, as an array of $v$ treatments arranged in $b$ columns of size $k$.

Output: $(t, T)$, the $T$ MRROSs and the treatment set $\phi$ for each MRROS.

1. If $k=2$ or $\alpha \geq\left\lfloor\frac{1}{2}(k+1)\right\rfloor$ record $t_{1}=b k$ and go to Stage 2. Otherwise calculate $t_{1}$ at Stage 1 according to Theorem 5 .

2. Set $t_{2}=b k$. For each $\phi$ with $\left\lfloor\frac{1}{2}(k+1)\right\rfloor \leq u \leq\left\lfloor\frac{1}{2} v\right\rfloor$, calculate $\rho(\phi)$. Store any $\phi$ with $\rho(\phi) \leq t_{1}$ in set $\Pi$. Identify any $\phi$ in $\Pi$ for which the SP-process generates at least one Type 1 RROS and for which the SP-process operating on any other member of $\Pi$ with lower $\rho(\phi)$ does not generate a Type 1 RROS. Update $t_{2}$ with the $\rho(\phi)$ value for any such sets.

3. If $t_{1}<t_{2}$ then $t=t_{1}$ and the MRROSs arise only from Stage 1 and are identified from Theorem 5. If $t_{2}<t_{1}$ then $t=t_{2}$ and the MRROSs arise only from Stage 2 . If $t_{2}=t_{1}$ then the MRROSs arise from both sources.

\section{Illustrations of the Method}

\subsection{Nearly Balanced Designs}

The approach of the paper is demonstrated by considering four designs, represented as $D_{1}, D_{2}, D_{3}, D_{4}$ in Fig. 5 , where $v=6, b=7, k=2$ with replications $r_{[1]}=r_{[2]}=3$, $r_{[i]}=2$ for $3 \leq i \leq 6$, such that each row in the figure denotes the seven blocks of the specified design. Although this example involves very small designs they demonstrate the difficulties that sometimes face the experimenter. Cheng and $\mathrm{Wu}$ (1981) show that these are the only non-isomorphic designs with these parameters that possess the property of being nearly balanced, i.e. the designs are binary, the treatment replication numbers differ by one at most and the pairwise treatment concurrences differ by one at most. 
Cheng and $\mathrm{Wu}$ (1981) derived $A$ - and $D$-efficiency lower bounds for the purpose of ranking the designs. Bailey (2007) gives three categories of design with block size 2 and $b=v+1$ and discusses the effect of observation loss on designs of each sort. The designs in Fig. 5 have Type 3 RROSs of the same cardinalities so it is interesting to look at the minimal partition numbers for each design.

Blocks

\begin{tabular}{|c|c|c|c|c|c|c|c|c|}
\hline & & 1 & 2 & 3 & 4 & 5 & 6 & 7 \\
\hline \multirow[t]{4}{*}{ Designs } & $\mathrm{D}_{1}$ & {$\left[\begin{array}{ll}1 & 2\end{array}\right]$} & [2 3] & {$\left[\begin{array}{ll}3 & 4\end{array}\right]$} & {$[45]$} & {$[56]$} & {$\left[\begin{array}{ll}6 & 1\end{array}\right]$} & {$\left[\begin{array}{ll}3 & 6]\end{array}\right.$} \\
\hline & $\mathrm{D}_{2}$ & {$\left[\begin{array}{ll}1 & 2\end{array}\right]$} & [2 3] & [3 4] & {$[45]$} & [5 6] & {$\left[\begin{array}{ll}6 & 1\end{array}\right]$} & {$[15]$} \\
\hline & $\mathrm{D}_{3}$ & {$\left[\begin{array}{ll}1 & 2\end{array}\right]$} & [2 3] & [3 4] & {$\left[\begin{array}{l}4 \\
5]\end{array}\right.$} & {$\left[\begin{array}{ll}5 & 1\end{array}\right]$} & {$\left[\begin{array}{ll}6 & 1\end{array}\right]$} & {$\left[\begin{array}{ll}3 & 6\end{array}\right]$} \\
\hline & $\mathrm{D}_{4}$ & {$\left[\begin{array}{ll}1 & 2\end{array}\right]$} & {$\left[\begin{array}{ll}2 & 3\end{array}\right]$} & {$\left[\begin{array}{ll}3 & 1\end{array}\right]$} & [45] & {$\left[\begin{array}{ll}5 & 6\end{array}\right]$} & {$\left[\begin{array}{ll}6 & 1\end{array}\right]$} & [46] \\
\hline
\end{tabular}

Fig 5: Four nearly balanced designs $D_{1}, D_{2}, D_{3}, D_{4}$,

Consider design $D_{4}$ which is the same as design $D$ given in Fig. 1; if the observation for treatment labelled 1 is lost from block 6 then blocks 1,2 and 3 contain all replicates of treatments labelled 1,2 and 3 and the remaining blocks contain all replicates of treatments labelled 4,5 and 6 so $D_{e}$ is disconnected. It follows that only six of the possible fifteen pairwise treatment contrasts are estimable, i.e. the loss of this single observation has had a very serious impact on the experiment. A similar situation arises if the single observation corresponding to treatment labelled 6 is lost from block 6 . However no more MRROSs of cardinality unity are generated, i.e. the loss of no further single observation gives a disconnected eventual design, showing that $(t, T)=(1,2)$ for design $D_{4}$.

The position is somewhat better for the other designs since the minimal partition numbers given by the procedure of the paper for $D_{1}, D_{2}$ and $D_{3}$ are $(t, T)=(2,20)$, $(t, T)=(2,24)$ and $(t, T)=(2,16)$ respectively. Cheng and $\mathrm{Wu}(1981)$ state that $D_{3}$ is the preferred design since it has higher $A$ - and $D$-efficiency lower bounds; it is clear from these minimal partition numbers that $D_{3}$ is also preferred on grounds of robustness.

\subsection{The Class of $(13,13,3)$ Designs}

A classic design is the partially balanced design with two association classes given by Bose (1963) in which thirteen treatments are arranged in thirteen blocks of size three, 
the design being based on a cyclic association scheme. The Bose design is isomorphic to a design $D_{5}$ which has initial block $[1,2,5]^{\prime}$ and the remaining twelve blocks are developed cyclically. It has high average efficiency factor of $2 / 3=0.6667$.

The authors are grateful to a referee for suggesting an examination of the class of equally replicated connected designs which have the same parameters as the Bose design, i.e. $(v, b, k)=(13,13,3)$. The aim is to investigate the relationship, if one exists, between efficiency and robustness. The DESIGN package (Soicher, 2011) for GAP (The GAP Group) shows that there are 479,741 non-isomorphic connected binary designs with these parameters. The Bose design is not most efficient in this class; design $D_{6}$ given in Fig. 6 has average efficiency factor 0.6695 and is the most efficient $(13,13,3)$ design.

Obviously all members of this class of $(13,13,3)$ designs have 13 simple Type 3 RROSs with cardinality 3 and the same distribution of Type 3 RROSs with higher cardinality, so it is required to compare the minimal partition numbers for this class. For all of these $(13,13,3)$ designs, when $u=1$ Theorem $5(\mathrm{i})$ gives $t_{1}=k-2+r_{[v]}=4$ and Corollary 1 gives $T_{1}=N_{[v]} r_{[v]}=39$. For design $D_{6}$, when $u \geq 2$ the SP-process identifies 39 Type 1 RROSs containing 5 observations. Therefore the MRROS cardinality and the number of sets for $D_{6}$ is $(t, T)=(4,39)$. These are the optimal minimal partition numbers for $(13,13,3)$ designs. Design $D_{5}$ also has minimal partition numbers $(t, T)=(4,39)$.

\section{Blocks}

\begin{tabular}{ccccccccccccc}
$\mathbf{1}$ & $\mathbf{2}$ & $\mathbf{3}$ & $\mathbf{4}$ & $\mathbf{5}$ & $\mathbf{6}$ & $\mathbf{7}$ & $\mathbf{8}$ & $\mathbf{9}$ & $\mathbf{1 0}$ & $\mathbf{1 1}$ & $\mathbf{1 2}$ & $\mathbf{1 3}$ \\
\hline 1 & 1 & 1 & 2 & 2 & 3 & 3 & 5 & 5 & 6 & 6 & 7 & 7 \\
2 & 4 & 6 & 4 & 8 & 4 & 10 & 8 & 9 & 8 & 10 & 9 & 11 \\
3 & 5 & 7 & 13 & 9 & 12 & 11 & 11 & 10 & 12 & 13 & 12 & 13 \\
\hline
\end{tabular}

Fig 6: Design $D_{6}$, the most efficient $(13,13,3)$ design

Let $D_{7}$ denote the least efficient $(13,13,3)$ design, not displayed here. $D_{7}$ has average efficiency factor 0.2759 and is less robust than designs $D_{5}, D_{6}$ as its minimal partition numbers are $(t, T)=(2,8)$. Thus an eventual design could be disconnected after losing only two observations, in 8 different ways; it could be disconnected after losing three observations in 296 different ways; and it could be disconnected after losing four observations in over 10,000 ways. Furthermore, it can be seen from additional designs in 
the $(13,13,3)$ class that ranking by robustness is not the same as ranking by efficiency. Fig. 7 gives a design $D_{8}$ with average efficiency factor $0.5435: D_{8}$ has a single MRROS of cardinality $t=2$ comprising one observation in each of blocks 3 and 4 , the removal of which separates treatments labelled 1,2 and 3 in the first three blocks from treatments labelled 4 to 13 in the remaining ten blocks. However, the cyclic design $D_{9}$ with initial block given by $[1,2,3]^{\prime}$ has average efficiency factor 0.4549 and minimal partition numbers $(t, T)=(4,104)$.

\section{$\underline{\text { Blocks }}$}

\begin{tabular}{ccccccccccccc}
$\mathbf{1}$ & $\mathbf{2}$ & $\mathbf{3}$ & $\mathbf{4}$ & $\mathbf{5}$ & $\mathbf{6}$ & $\mathbf{7}$ & $\mathbf{8}$ & $\mathbf{9}$ & $\mathbf{1 0}$ & $\mathbf{1 1}$ & $\mathbf{1 2}$ & $\mathbf{1 3}$ \\
\hline 1 & 1 & 1 & 3 & 4 & 4 & 4 & 5 & 6 & 7 & 8 & 9 & 10 \\
2 & 2 & 2 & 6 & 5 & 6 & 11 & 12 & 7 & 8 & 9 & 10 & 11 \\
3 & 3 & 5 & 8 & 7 & 13 & 12 & 13 & 9 & 10 & 11 & 12 & 13
\end{tabular}

Fig 7: Design $D_{8}$, a $(13,13,3)$ design with poor robustness

These results for the five designs are summarised in Table 1, ranking by efficiency. Minimal partition numbers are given as well as $\left(r_{[v]}, N_{[v]}\right)$, the corresponding numbers for Type 3 RROSs. Also given are the probabilities $p_{\ell}$ that not all treatment contrasts are estimable, conditional on the loss of $\ell$ observations, for $\ell=1,2,3,4$. The table shows that ranking of designs by robustness is different from ranking of designs by efficiency.

\begin{tabular}{ccrrcccc} 
Design & AEF & $(t, T)$ & $\left(r_{[v]}, N_{[v]}\right)$ & $p_{1}$ & $p_{2}$ & $p_{3}$ & $p_{4}$ \\
\hline$D_{6}$ & 0.6695 & $(4,39)$ & $(3,13)$ & 0 & 0.0000 & 0.0014 & 0.0062 \\
$D_{5}$ & 0.6667 & $(4,39)$ & $(3,13)$ & 0 & 0.0000 & 0.0014 & 0.0062 \\
$D_{8}$ & 0.5435 & $(2,1)$ & $(3,13)$ & 0 & 0.0013 & 0.0055 & 0.0138 \\
$D_{9}$ & 0.4549 & $(4,104)$ & $(3,13)$ & 0 & 0.0000 & 0.0014 & 0.0070 \\
$D_{7}$ & 0.2759 & $(2,8)$ & $(3,13)$ & 0 & 0.0108 & 0.0338 & 0.0705 \\
\hline
\end{tabular}

Table 1: Average efficiency factor (AEF) and minimal partition numbers for five designs

\subsection{Sequences of Resolvable $(9,3 \mathrm{r}, 3)$ Designs}

Clatworthy (1973) has given a sequence of resolvable group divisible designs for $v=9$ and $k=3$, such that nine treatments are arranged in $3 r$ blocks, where $r \geq 5$ denotes the number of replicates for a design in the sequence. For each design the treatments divide 
into three groups of size three such that treatments within the same group have concurrence $\lambda_{1}=r-3$ and those in different groups have concurrence $\lambda_{2}=1$; furthermore all designs are the regular subtype since $r>\lambda_{1}$ and $r k=3 r>9=v \lambda_{2}$. The construction of these designs is based on four sets of blocks designated A1, A2, A3, A4 given in Fig. 8. Each design is a single copy of A1, A2 and A3, together with $r-3$ copies of A4. Clatworthy denoted members of the sequence for $r=5, \ldots, 10$ by R59, R60, R61, R63, R64, R66; the average efficiency factors of these designs are $0.7386,0.7143,0.6857,0.6563,0.6275$ and 0.6000 respectively.

\begin{tabular}{|c|c|c|c|c|c|c|c|c|c|c|c|}
\hline \multicolumn{3}{|c|}{$\underline{\text { Blocks A1 }}$} & \multicolumn{3}{|c|}{$\underline{\text { Blocks A2 }}$} & \multicolumn{3}{|c|}{$\underline{\text { Blocks A3 }}$} & \multicolumn{3}{|c|}{$\underline{\text { Blocks A4 }}$} \\
\hline 1 & 2 & 3 & 1 & 2 & 3 & 1 & 2 & 3 & 1 & 2 & 3 \\
\hline 1 & 4 & 7 & 1 & 2 & 3 & 1 & 2 & 3 & 1 & 2 & 3 \\
\hline 2 & 5 & 8 & 6 & 4 & 5 & 5 & 6 & 4 & 4 & 5 & 6 \\
\hline 3 & 6 & 9 & 8 & 9 & 7 & 9 & 7 & 8 & 7 & 8 & 9 \\
\hline
\end{tabular}

Fig 8: Constituent block sets for sequences of resolvable designs

An alternative sequence of resolvable designs with the same parameters can be found using copies of A1, A2, A3, A4 which are nearly equally replicated; six designs corresponding to the Clatworthy group divisible designs are given, respectively, by A1, A2, A3, A4 copied $(2,1,1,1),(2,2,1,1),(2,2,2,1),(2,2,2,2),(3,2,2,2)$ and $(3,3,2,2)$ times. These designs are, respectively, $D_{10}, D_{11}, D_{12}, D_{13}, D_{14}$ and $D_{15}$; they are not partially balanced but they are nearly balanced, except for $D_{13}$ which is balanced, and they have average efficiency factors $0.7386,0.7407,0.7453,0.7500,0.7467$ and 0.7467 respectively.

\begin{tabular}{ccccccccc}
$r$ & $b k$ & $\left(r_{[v]}, N_{[v]}\right)$ & Design & AEF & $(t, T)$ & Design & AEF & $(t, T)$ \\
\hline 5 & 45 & $(5,9)$ & $R 59$ & 0.7386 & $(6,45)$ & $D 10$ & 0.7386 & $(6,45)$ \\
6 & 54 & $(6,9)$ & $R 60$ & 0.7143 & $(7,54)$ & $D 11$ & 0.7407 & $(7,54)$ \\
7 & 63 & $(7,9)$ & $R 61$ & 0.6857 & $(8,63)$ & $D 12$ & 0.7453 & $(8,63)$ \\
8 & 72 & $(8,9)$ & $R 63$ & 0.6563 & $(9,75)$ & $D 13$ & 0.7500 & $(9,72)$ \\
9 & 81 & $(9,9)$ & $R 64$ & 0.6275 & $(9,3)$ & $D 14$ & 0.7467 & $(10,81)$ \\
10 & 90 & $(10,9)$ & $R 66$ & 0.6000 & $(9,3)$ & $D 15$ & 0.7467 & $(11,90)$ \\
\hline
\end{tabular}

Table 2: AEF and robustness measures for two design sequences

This alternative use of the blocks in Fig. 8 gives a more efficient sequence of designs and it is interesting to ask which sequence of designs is the more robust. For each 
value of $r$ Table 2 gives the $\left(r_{[v]}, N_{[v]}\right)$ and $(t, T)$ numbers for the corresponding pair of designs. These results show that each of the larger group divisible designs given by Clatworthy has weaker robustness properties than the alternative design of the same size. Furthermore, each sequence can be extended in an obvious way beyond $r=10$ and it is straightforward to show that the pattern of these results is continued.

The procedure was performed on these designs using 64 bit Matlab version 7.12.0 (R2011a) executed on a $2.66 \mathrm{GHz}$ Intel ${ }^{\mathrm{R}}$ Core $^{\mathrm{TM}_{2}}$ Duo processor. The time taken to complete the procedure for a single design varied between 0.61 and 0.96 seconds. In each case the minimal partition numbers were identified and the MRROSs listed along with the corresponding treatment separation, $\phi, \phi^{c}$. The speed of the process is in marked contrast to the time that would be required for a search for Type 1 RROSs through the

prohibitively large number of observation sets, viz. $\sum_{i=1}^{t}\left(\begin{array}{c}b k \\ i\end{array}\right)=\sum_{i=1}^{9}\left(\begin{array}{c}90 \\ i\end{array}\right) \simeq 8 \times 10^{11}$ for R66, required by the search routine of Godolphin (2006).

\subsection{Comparison of Large Two-Resolvable Designs}

Nguyen (1994) proposed two alternative two-resolvable designs for an experiment involving 210 observations to compare 21 timber species in 35 blocks of size 6 . One suggested design comprises 5 copies of the PBIB[2] design, designated T69 by Clatworthy (1973), which is based on a triangular association scheme. Nguyen remarked that this design is likely to have poor properties since the concurrences are not evenly distributed, with some pairs of treatments appearing together in 5 blocks and others not appearing together in any block. Nguyen constructed an alternative two-resolvable design in which each pair of treatments occurred together in either two or three blocks. It is interesting to consider whether these two designs have the same minimal partition numbers. Since both designs are very large this provides a real test for the treatment separation method. Use of the procedure gives the minimal partition numbers $(t, T)=(14,210)$ in both cases, so neither design is preferable on this criterion. The procedure required approximately 100 minutes to complete for each design, using the same Matlab program and processor as in $\S 3.3$.

\section{Acknowledgements}

The authors are grateful to two referees for suggestions for improving the paper. 


\section{References}

Angelis, L. 2003. An evolutionary algorithm for A-optimal incomplete block designs. J. Statist. Computation Simul. 73, 753-771.

Bailey, R.A. 2007. Designs for two-colour microarray experiments. Appl. Statist. 56, 365-394.

Bate, S.T., Godolphin, E.J., Godolphin, J.D. 2008. Choosing cross-over designs when few subjects are available. Comput. Statist. Data Anal. 52, 1572-1586.

Bose, R.C. 1963. Combinatorial properties of partially balanced designs and association schemes. Sankya Ser. A, 25, 109-136.

Cheng, C-S., Wu, C-F. 1981. Nearly balanced incomplete block designs. Biometrika, 68, 493-500.

Clatworthy, W.H. 1973. Tables of two-associate-class partially balanced designs. Nat. Bureau Stand. Appl. Math. Ser. 63.

Cochran, W.G. and Cox, G.M. 1957. Experimental Designs. 2nd ed. New York: Wiley.

Dey, A., Srivastava, R., Parsad, R. 2001. Robustness of block designs for diallel crosses against missing observations. J. Ind. Soc. Ag. Statist. 54, 376-384.

The GAP Group, 2013. GAP - Groups, Algorithms and Programming, version 4.6.4; 2013. (http://www.gap-system.org)

Godolphin, J.D., 2004. Simple pilot procedures for the avoidance of disconnected experimental designs. Applied Statistics 53, 133-147.

Godolphin, J.D., 2006. The specification of rank reducing observation sets in experimental design. Comput. Statist. Data Anal. 51, 1862-1874.

Godolphin, J.D., 2013. On the connectivity problem for $m$-way designs. J. Statist. Th. Prac., 7, (to appear)

Godolphin, J.D., Warren, H.R., 2011. Improved conditions for the robustness of binary block designs against the loss of whole blocks. J. Statist. Plan. Inf. 141, 3498-3505.

John, J.A., Whitaker, D. and Triggs, C.M. 1993. Construction of cyclic designs using integer programming. J. Statist. Planng Inf. 36, 357-366.

Jones, B., Eccleston, J.A. 1980. Exchange and interchange procedures to search for optimal designs. J. R. Statist. Soc. Ser B, 42, 238-243.

Mitchell, T.J. 1974. Computer construction of D-optimal first-order designs. Technometrics 16, 211-220. 
Nguyen, N-K. 1994. Construction of optimal block designs by computer. Technometrics 36, 300-307.

Nguyen, N-K., Miller, A.J. 1992. A review of some exchange algorithms for constructing discrete D-optimal designs. Comp. Statist. Data Anal. 14, 489-498.

Soicher, L.H. 2011. The DESIGN package for GAP, Version 1.6, 2011.

http://designtheory.org/software/gap_design/

Theil, H., 1965. The analysis of disturbances in regression analysis. J. Amer. Statist. Assoc. 60, 1067-1079.

Whitaker, D., Triggs, C.M., John, J.A. 1990. Construction of block designs using mathematical programming. J. R. Statist. Soc. Ser B, 52, 497-503.

Yates, F. 1933. The analysis of replicated experiments when the field results are incomplete. Empire J. Experimental Agriculture 1, 129-142. 